\title{
GRB 060313: A NEW PARADIGM FOR SHORT-HARD BURSTS?
}

\author{
Peter W. A. Roming, ${ }^{1}$ Daniel Vanden Berk, ${ }^{1}$ Valentin Pal'shin, ${ }^{2}$ Claudio Pagani, ${ }^{1}$ Jay Norris, ${ }^{3}$ Pawan Kumar, ${ }^{4}$ \\ Hans Krimm, ${ }^{3,5}$ Stephen T. Holland, ${ }^{3,5}$ Caryl Gronwall, ${ }^{1}$ Alex J. Blustin, ${ }^{6}$ Bing Zhang, ${ }^{7}$ Patricia Schady, ${ }^{1,6}$ \\ Takanori Sakamoto, ${ }^{3}$ Julian P. Osborne, ${ }^{8}$ John A. Nousek, ${ }^{1}$ Frank E. Marshall, ${ }^{3}$ Peter Mészáros, ${ }^{1,9}$ \\ Sergey V. Golenetskit, ${ }^{2}$ Neil Gehrels, ${ }^{3}$ Dmitry D. Frederiks, ${ }^{2}$ Sergio Campana, ${ }^{10}$ \\ David N. Burrows, ${ }^{1}$ Patricia T. Boyd, ${ }^{3}$ Scott Barthelmy, ${ }^{3}$ and R. L. Aptekar ${ }^{2}$ \\ Received 2006 April 28; accepted 2006 July 27
}

\begin{abstract}
We report the simultaneous observations of the prompt emission in the gamma-ray and hard X-ray bands by the Swift BAT and the Konus-Wind instruments of the short-hard burst, GRB 060313. The observations reveal multiple peaks in both the gamma-ray and hard X-ray bands suggesting a highly variable outflow from the central explosion. We also describe the early-time observations of the X-ray and UV/optical afterglows by the Swift XRT and UVOT instruments. The combination of the X-ray and UV/optical observations provides the most comprehensive light curves to date of a short-hard burst at such an early epoch. The afterglows exhibit complex structure with different decay indices and flaring. This behavior can be explained by the combination of a structured jet, radiative loss of energy, and decreasing microphysics parameters occurring in a circumburst medium with densities varying by a factor of approximately two on a length scale of $10^{17} \mathrm{~cm}$. These density variations are normally associated with the environment of a massive star and inhomogeneities in its windy medium. However, the mean density of the observed medium $\left(n \sim 10^{-4} \mathrm{~cm}^{3}\right)$ is much less than that expected for a massive star. Although the collapse of a massive star as the origin of GRB 060313 is unlikely, the merger of a compact binary also poses problems for explaining the behavior of this burst. Two possible suggestions for explaining this scenario are that some short bursts may arise from a mechanism that does not invoke the conventional compact binary model, or that soft late-time central engine activity is producing UV/optical but no X-ray flaring.
\end{abstract}

Subject headings: gamma rays: bursts

Online material: color figures

\section{INTRODUCTION}

Gamma-ray bursts (GRBs) are generally classified into one of two categories: long-soft and short-hard (Kouveliotou et al. 1993). ${ }^{11}$ Short GRBs are bursts with $T_{90}<2 \mathrm{~s}$ (where $T_{90}$ is the time in which the cumulative counts above background are between $5 \%$ and $95 \%$ of the total) and a hardness ratio that is typically larger than the one of long GRBs. Short bursts also exhibit an initial spike with insignificant spectral evolution at energies greater than $25 \mathrm{keV}$ (Norris \& Bonnell 2006). From $25 \%$ to $30 \%$ of all

\footnotetext{
1 Department of Astronomy and Astrophysics, Pennsylvania State University, 525 Davey Lab, University Park, PA 16802; roming@astro.psu.edu.

2 Ioffe Physico-Technical Institute, 26 Polytekhnicheskaya, St. Petersburg 194021, Russian Federation.

3 NASA Goddard Space Flight Center, Greenbelt, MD 20771.

4 Department of Astronomy, University of Texas at Austin, 1 University Station, C1400 Austin, TX 78712-0259.

${ }^{5}$ Universities Space Research Association, 10227 Wincopin Circle, Suite 500, Columbia, MD 21044.

6 Mullard Space Science Laboratory, University College London, Holmbury St. Mary, Dorking, Surrey RH5 6NT, UK.

7 Department of Physics, University of Nevada, 4505 Maryland Parkway, Las Vegas, NV 89154-4002.

8 Department of Physics and Astronomy, University of Leicester, University Road, Leicester LE1 7RH, UK.

9 Department of Physics, Pennsylvania State University, 104 Davey Lab, University Park, PA 16802.

10 INAF-Osservatorio Astronomico di Brera, via E. Bianchi 46, I-23807 Merate (LC), Italy.

11 Two additional classes of bursts have been proposed: an intermediate class (Horváth 1998; Mukherjee et al. 1998; Horváth et al. 2006), which falls between the short- and long-burst classes with respect to $T_{90}$ and has a hardness ratio softer than long bursts, and a very short class (Cline et al. 2005) that has a very hard spectrum and $T_{90} \leq 100 \mathrm{~ms}$.
}

GRBs in the Burst and Transient Source Experiment (BATSE) catalog (Fishman et al. 1994) are short-hard bursts (SHBs; Kouveliotou et al. 1993), while $12 \% \pm 4 \%$ of the Swift (Gehrels et al. 2004) sample are SHBs. It has been proposed that SHBs are the result of a compact binary merger such as a double neutron star (DNS; Paczyński 1986; Eichler et al. 1989; Narayan et al. 1992; Fryer et al. 1999; Rosswog et al. 2003; Lee et al. 2005; Panaitescu 2006) or a neutron star and a black hole (NS-BH; Lattimer \& Schramm 1976; Paczyński 1991; Narayan et al. 1992; Mochkovitch et al. 1993; Fryer et al. 1999; Lee et al. 2005; Panaitescu 2006). Since the natal kick of a neutron star is $\sim 200$ $1000 \mathrm{~km} \mathrm{~s}^{-1}$, and since the typical life time of compact binaries is $\sim 0.1-1$ Gyr (Panaitescu 2006), a compact binary could wander anywhere from $20 \mathrm{kpc}$ to $1 \mathrm{Mpc}$ in the time it takes for the binary to merge. At these large distances from the neutron star's stellar nursery, a lower density external medium is expected.

Understanding and testing these different short GRB models has been difficult due to the large time delay in localizing the burst afterglows. The Interplanetary Network (IPN; Hurley et al. 2005) has triangulated a few short GRBs on timescales of days (Bloom et al. 2006); however, on these timescales, the afterglow has long since faded. Since the launch of the Swift satellite, $11 \mathrm{SHBs}^{12}$ have been localized in under an hour. Four of the

12 It has been noted in the literature that there have been other welllocalized short bursts that have soft spectra (Bloom et al. 2006). Recent work (Levan et al. 2006; Sakamoto et al. 2006) has suggested that Swift and HETE-2 short bursts have softer spectra than the BATSE bursts. They find that the hardness ratio is between that of the BATSE short and long GRBs, but the Swift short bursts are typically harder than the long ones. Hereafter, we assume the Swift and HETE-2 short bursts fit into the hard classification scheme and refer to short bursts as those bursts with short-hard spectra. 
11 bursts, GRBs 050202 (Tueller et al. 2005), 050906 (Krimm et al. 2005), 050925 (Holland et al. 2005), and 051105A (Mineo et al. 2005), were localized in the hard X-ray band by the Swift Burst Alert Telescope (BAT; Barthelmy et al. 2005a) but with no corresponding X-ray, optical, or radio detections; three additional bursts, GRBs 050509B (Gehrels et al. 2005; Bloom et al. 2006), 050813 (Retter et al. 2005; Morris et al. 2005), and 051210 (La Parola et al. 2006), were localized by the BAT with corresponding Swift X-ray Telescope (XRT; Burrows et al. 2005b) detections but no optical or radio detections; the remaining four bursts, GRBs 050724 (Barthelmy et al. 2005b; Berger et al. 2005; Campana et al. 2006; Grupe et al. 2006; Vaughan et al. 2006), 051221A (Parsons et al. 2005; Burrows et al. 2005a, 2006; Grupe et al. 2005; Roming et al. 2005a; Soderberg et al. 2006), 051227 (L. M. Barbier et al. 2006, in preparation), and 060313 (Pagani et al. 2006b), were localized by the BAT and XRT with corresponding Swift Ultraviolet/Optical Telescope (UVOT; Roming et al. 2005b) and/or ground-based detections. Additional rapid observations of GRBs 050709 (Fox et al. 2005; Villasenor et al. 2005; Covino et al. 2006) and 060121 (Arimoto et al. 2006; Levan et al. 2006) were made with the High Energy Transient Explorer (HETE-2). These rapid observations have broadened our understanding of short GRBs and provided strong evidence that the origin of short bursts is different from that of long bursts and is consistent with the merger of compact objects (Gehrels et al. 2005; Barthelmy et al. 2005b; Fox et al. 2005; Villasenor et al. 2005).

However, the data for GRB 060313 indicates that there are problems with the model for a compact binary merger, at least for this burst. Two possible explanations for the observed behavior are that some short bursts may be produced by a different mechanism than that invoked by the standard compact binary model, or that late-time central engine activity is generating late internal shocks from which low-energy (UV/optical) flares arise. Here we present the broadband nature of the short GRB 060313 and its afterglow based on Swift BAT, XRT, and UVOT, as well as KonusWind (Aptekar et al. 1995) data. This is only the fifth short burst with a reported optical afterglow and the first with UV detections and optical flaring. It is also the hardest burst in the Swift sample. In addition, the optical and UV detections made by the UVOT of this burst are the earliest $(<80 \mathrm{~s})$ optical/UV detections of a short burst to date. The combination of the UVOT data with the BAT, Konus, and XRT data provides the most comprehensive light curves to date of a SHB at such an early epoch and affords a unique opportunity to probe the mechanism behind this short GRB.

\section{OBSERVATIONS AND DATA ANALYSIS}

On 2006 March 13, at $00: 12: 06.484$ UT, the BAT triggered on GRB 060313 (Pagani et al. 2006b). The timing analysis hereafter is referenced from the BAT trigger time $\left(T_{0}\right)$. The initial BAT light curve has two slightly overlapping peaks with a total duration of $\sim 1 \mathrm{~s}$. The peak count rate was approximately 90,000 counts $\mathrm{s}^{-1}(15-350 \mathrm{keV})$ occurring at $T_{0}+0.5 \mathrm{~s}$.

The UVOT began a finding chart exposure $78 \mathrm{~s}$ after the trigger. The afterglow was discovered during ground processing. The XRT began its autonomous sequence of observations of the GRB field at $00: 13: 24$ UT, $79 \mathrm{~s}$ after the BAT trigger. The XRT onboard centroiding algorithm could not converge on a source in the image, but a fading source was detected in the ground-processed data (Pagani et al. 2006a). The first ground-based detection was made at $01: 28$ UT with the VLT and FORS2 Telescopes (Levan $\&$ Hjorth 2006). No radio source was detected at the VLT position (Soderberg \& Frail 2006).

Hereafter, we adopt the notation $F(\nu, t) \propto t^{-\alpha} \nu^{-\beta}$ for the afterglow flux as a function of time, where $\nu$ is the frequency of

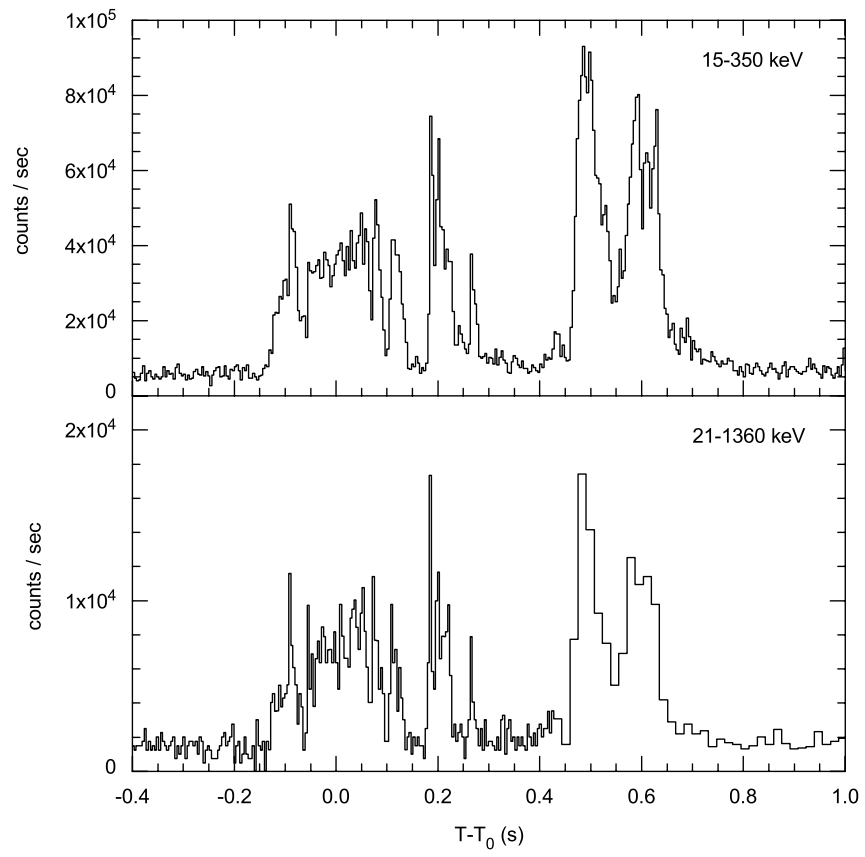

FIG. 1.-BAT and Konus-Wind light curves. The BAT $15-350 \mathrm{keV}$ light curve (top panel) has at least 20 statistically significant peaks with FWHMs in the 5$15 \mathrm{~ms}$ range. The average BAT error is 1250 counts s${ }^{-1}$. The Konus-Wind 21$1360 \mathrm{keV}$ light curve (bottom panel) also exhibits several multipeaked pulses, with a total duration of approximately $0.8 \mathrm{~s}$.

the observed flux, $t$ is the time post trigger, $\beta$ is the spectral index, which is related to the photon index $\Gamma(\beta=\Gamma-1)$, and $\alpha$ is the temporal decay slope. We also adopt the convention $q_{x}=$ $10^{x} q$ in cgs units. A flat cosmological-constant-dominated cosmology with the parameter values $H_{0}=70 \mathrm{~km} \mathrm{~s}^{-1} \mathrm{Mpc}^{-1}, \Omega_{M}=$ 0.3 , and $\Omega_{\Lambda}=0.7$ is assumed.

\subsection{BAT Analysis}

Ground analysis (Markwardt et al. 2006) of the BAT data determined that $T_{90}$ for the $15-350 \mathrm{keV}$ band is $0.7 \pm 0.1 \mathrm{~s}$ (estimated error includes the systematics) with a fluence of $(1.13 \pm 0.05) \times 10^{-6} \mathrm{ergs} \mathrm{cm}^{-2}$ in the $15-150 \mathrm{keV}$ band. The $1 \mathrm{~s}$ peak photon flux measured from $T_{0}-0.124 \mathrm{~s}$ in the $15-150 \mathrm{keV}$ band is $12.1 \pm 0.4$ photons $\mathrm{cm}^{-2} \mathrm{~s}^{-1}$. All BAT errors are at the $90 \%$ confidence level.

In the $15-350 \mathrm{keV}$ light curve (Fig. 1) at least 20 statistically significant peaks with FWHMs in the 5-15 ms range (Barthelmy et al. 2006) can be seen. There is no periodic structure in the light curve for at least the first $100 \mathrm{~s}$. There is also no evidence of extended emission in the $T_{0}+1$ to $T_{0}+300 \mathrm{~s}$ range at an upper limit of 0.001 counts detector ${ }^{-1} \mathrm{~s}^{-1}(3 \sigma)$. This is consistent with an upper limit on the flux ratio between the initial peak and the peak of any potential extended emission of $\sim 2000$. SHBs 050724 and 051227 had flux ratios of 46 and $\sim 10$, respectively (Barthelmy et al. 2006).

A four-channel light curve (Fig. 2) reveals that the burst is a short hard burst. A lag analysis confirmed the burst to cleanly reside in the short hard burst class (Fig. 3 in Norris \& Bonnell 2006). The measured lags are $0.8 \pm 0.6 \mathrm{~ms}[(50-100 \mathrm{keV}) /$ $(15-25 \mathrm{keV})]$ and $0.3 \pm 0.7 \mathrm{~ms}[(100-350 \mathrm{keV}) /(25-50 \mathrm{keV})]$.

\subsection{Konus-Wind Analysis}

Konus-Wind $(\mathrm{K}-\mathrm{W})$ triggered on GRB 060313 at $T_{0}(\mathrm{~K}-\mathrm{W})=$ $00: 12: 06.354$ UT (Golenetskii et al. 2006). It was detected by the S1 detector, which observes the south ecliptic hemisphere; 


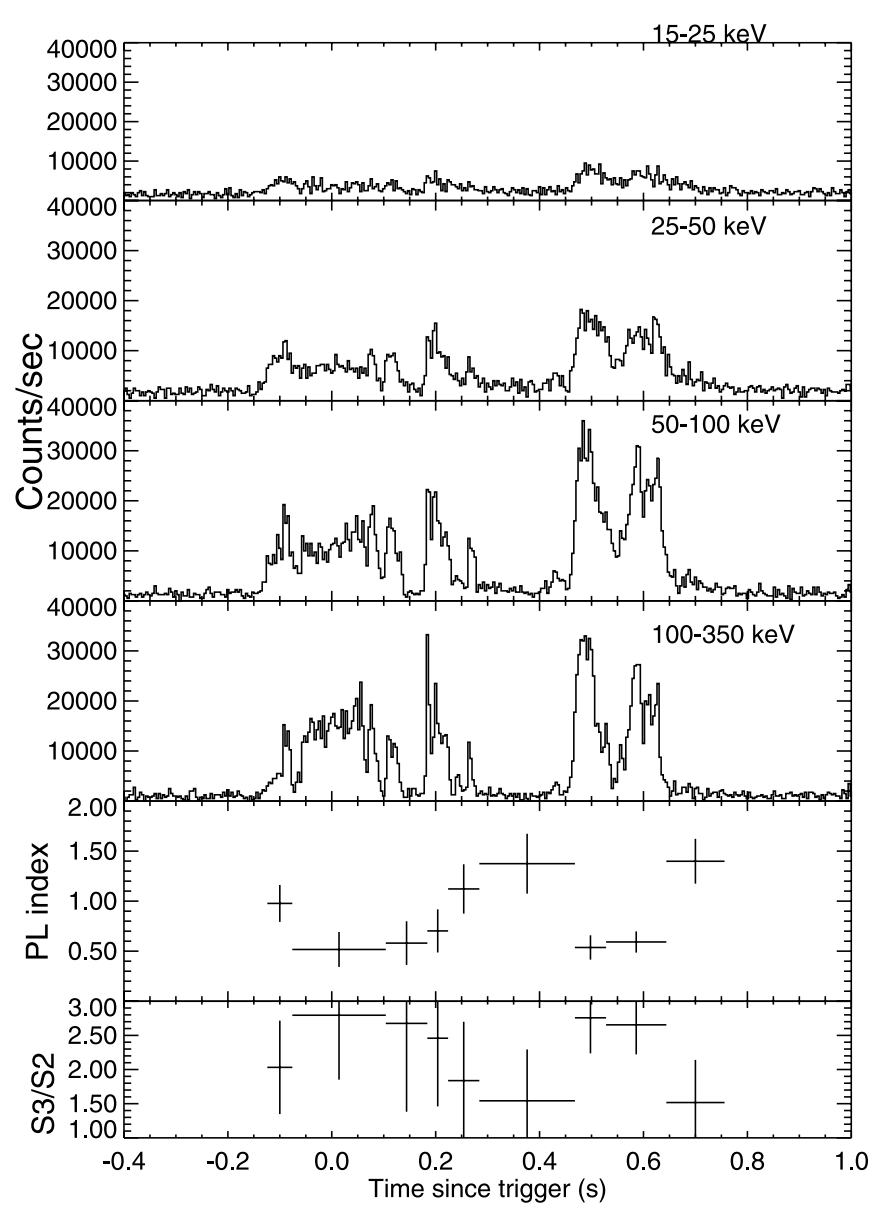

FIg. 2.-BAT four channel $(15-25,25-50,50-100$, and $100-350 \mathrm{keV})$ light curves. The average errors are $655,670,600$, and 530 counts s ${ }^{-1}$, respectively. The fifth panel from the top shows the photon power-law index for a simple power-law fit. The bottom panel shows the fluence hardness ratio $\mathrm{S} 3 / \mathrm{S} 2$ between the $50-100 \mathrm{keV}$ and $25-50 \mathrm{keV}$ bands, where the fluence was calculated based on a power-law fit. The hardness ratio for the time integrated spectral fit is 2.43 .

the incident angle was $57^{\circ} .9$. The propagation delay from Swift to Wind is $0.045 \mathrm{~s}$ for this GRB; therefore, correcting for this factor, one sees that the K-W trigger time corresponds to $T_{0}-0.085 \mathrm{~s}$.

The GRB time history was recorded in three energy ranges: G1 (21-83 keV), G2 (83-360 keV), and G3 (360-1360 keV). Thanks to the high intensity of the burst, the $\mathrm{K}-\mathrm{W}$ reserve system (so-called nonius) was triggered providing $2 \mathrm{~ms}$ resolution record in the G2 and G3 ranges of the entire burst [up to $T_{0}(\mathrm{~K}-\mathrm{W})+$ $0.762 \mathrm{~s}]$. Five spectra in 101 channels were accumulated during the burst (the total number of spectra accumulated during the trigger record is 64). The light curve (Fig. 1) shows several multipeaked pulses with a total duration of approximately $0.8 \mathrm{~s}$. As with the BAT multichannel light curve, the three channel Konus light curves (Fig. 3) illustrate that the burst is a short-hard burst.

\subsection{XRT Analysis}

An uncataloged, fading source was discovered during ground analysis (Pagani et al. 2006a) of the XRT data. The X-ray afterglow was observed in Windowed Timing (WT) mode for $26 \mathrm{~s}$. As the source faded the XRT switched into photon counting (PC) mode, thus providing two-dimensional spatial information (for a complete description of the XRT modes see Hill et al. 2004). To avoid pile-up in the PC data during the first orbit of observations, the XRT light curve was extracted in the energy

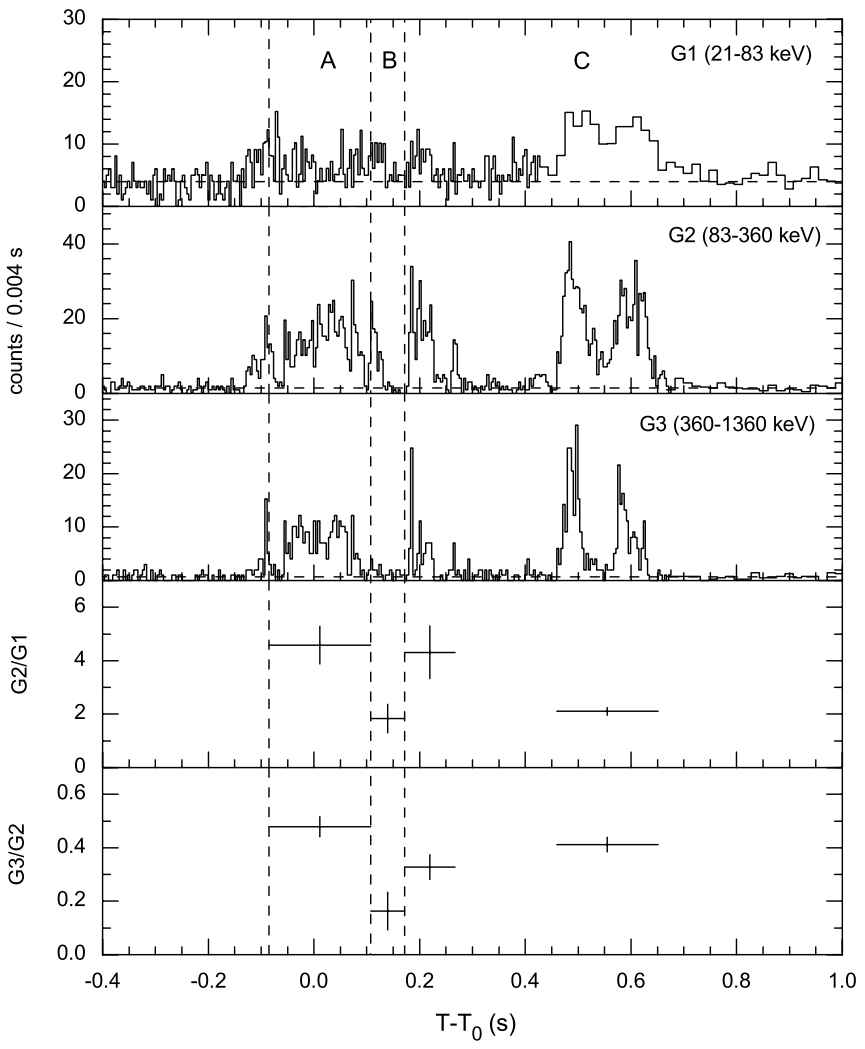

FIG. 3.-Konus-Wind light curve of GRB 060313 in three energy bands. The two bottom panels show the hardness ratios G2/G1 and G3/G2, which demonstrates significant spectral evolution during the burst. The dashed vertical lines denote the boundaries of the three intervals over which we extracted the spectra in $\S 2.4$.

range $0.2-10.0 \mathrm{keV}$ using an annulus centered on the afterglow position with an inner radius of 2 pixels and applying a PSF correction. The X-ray light curve (see Fig. 4) during the first orbit of observation manifested variability, with spikes and fluctuations superimposed on the overall fading behavior. The initial decay slope was $\alpha=1.25 \pm 0.15$. The light-curve profile becomes smoother in the later orbits $(>4200 \mathrm{~s}$ after the BAT trigger), and the decay curve can be fitted by a power law with index

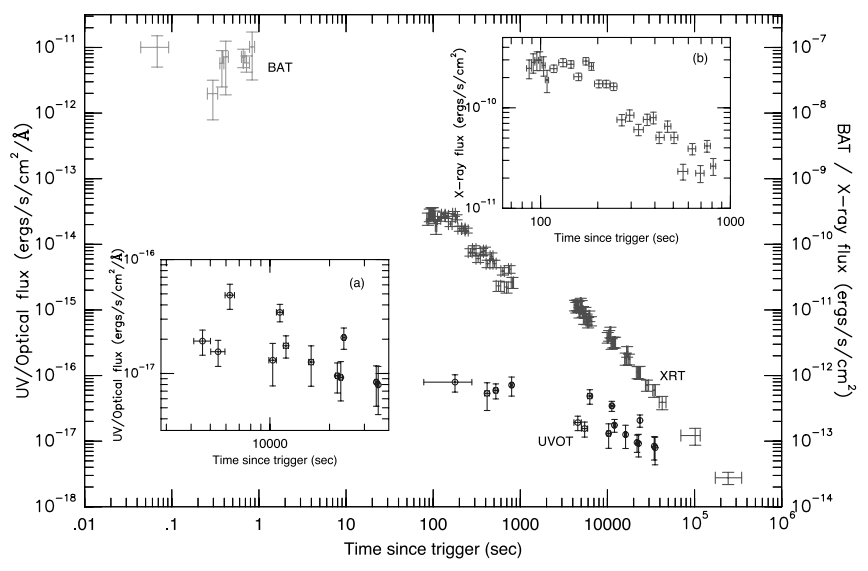

FIG. 4.-Combined BAT, XRT, and UVOT light curves. The BAT values are extrapolated to the XRT $0.3-10 \mathrm{keV}$ energy range, and the UVOT values are adjusted to the $U$ band. The two insets are provided to show ( $a$ ) the flaring of the UVOT and (b) X-ray light curves after $3000 \mathrm{~s}$ and during the first $1000 \mathrm{~s}$, respectively. [See the electronic edition of the Journal for a color version of this figure.] 
TABLE 1

Swift UVOT Photometry of the Afterglow of GRB 060313

\begin{tabular}{|c|c|c|c|c|c|}
\hline $\begin{array}{c}t \\
(\mathrm{~s})\end{array}$ & $\begin{array}{l}\Delta t \\
(\mathrm{~s})\end{array}$ & Filter & Magnitude & Error & $\begin{array}{c}\text { Adjusted } U \text {-Band } \\
\text { Magnitude }\end{array}$ \\
\hline 178. & 200 & $V$ & 19.56 & 0.32 & 19.06 \\
\hline ............ & 50 & $U$ & 19.49 & 0.49 & 19.49 \\
\hline $524 \ldots \ldots \ldots \ldots \ldots$ & 50 & White & 19.07 & 0.28 & 19.38 \\
\hline $795 \ldots \ldots \ldots \ldots$ & 50 & $U$ & 19.17 & 0.35 & 19.17 \\
\hline $4568 \ldots \ldots \ldots \ldots \ldots$ & 886 & $B$ & 20.85 & 0.27 & 20.60 \\
\hline $5476 \ldots \ldots \ldots \ldots \ldots$ & 900 & UVW2 & 20.20 & 0.28 & 20.83 \\
\hline $6280 \ldots \ldots \ldots \ldots \ldots$ & 684 & $V$ & 20.09 & 0.27 & 19.59 \\
\hline $10328 \ldots \ldots \ldots \ldots$ & 900 & UVM2 & 20.53 & 0.44 & 21.02 \\
\hline $11235 \ldots \ldots \ldots \ldots$ & 900 & UVW1 & 19.66 & 0.19 & 19.97 \\
\hline 12052. & 708 & $U$ & 20.70 & 0.24 & 20.70 \\
\hline $16147 .$. & 886 & $B$ & 21.31 & 0.42 & 21.06 \\
\hline $21890 \ldots \ldots \ldots \ldots$ & 900 & UVM2 & 20.87 & 0.32 & 21.36 \\
\hline $22797 \ldots \ldots \ldots \ldots$ & 900 & UVW1 & 21.09 & 0.41 & 21.40 \\
\hline $23618 \ldots \ldots \ldots \ldots . . . .$. & 716 & $U$ & 20.52 & 0.23 & 20.52 \\
\hline $34426 \ldots \ldots \ldots \ldots . . . .$. & 900 & UVW1 & 21.19 & 0.42 & 21.50 \\
\hline $35217 \ldots \ldots \ldots \ldots$ & 658 & $U$ & 21.56 & 0.49 & 21.56 \\
\hline
\end{tabular}

of $1.46 \pm 0.08$. In addition, the flux at these later times is larger by a factor of $\sim 4$ compared with the extrapolation of the earlier $\mathrm{X}$-ray light curve. The X-ray light curve is shown in Figure 4, with the count rate converted into $0.2-10.0 \mathrm{keV}$ unabsorbed flux using the best spectral model fit.

The X-ray spectrum from the PC data was extracted in the $0.3-10.0 \mathrm{keV}$ energy range, with a binning of at least 20 counts per energy bin, using the latest versions of the ancillary and response matrix files. The PC spectrum of the first orbit of observation was extracted excluding the central 2 pixels to avoid pile up. The spectrum can be fitted by a simple absorbed power law, yielding a photon index of $1.53 \pm 0.10$ and an absorption column density $N_{\mathrm{H}}$ consistent with the Galactic value of $4.7 \times 10^{20} \mathrm{~cm}^{-2}$ (Dickey \& Lockman 1990). The spectrum softens during the later orbits: the best power-law model fit of the afterglow spectrum extracted from 4.1 to $24 \mathrm{ks}$ after the GRB trigger yields a photon index of $1.96 \pm 0.09$, fixing the $N_{\mathrm{H}}$ at the Galactic value.

\subsection{UVOT Analysis}

The Swift spacecraft slewed promptly when the BAT detected GRB 060313, and UVOT began imaging the field shortly after the BAT trigger. The UVOT took 44 exposures of the field between 78 and $67,783 \mathrm{~s}$ after the BAT trigger. For the majority of these exposures the afterglow was too faint to be detected. The UVOT photometry that is used in this paper is presented in Table 1. The UVW2 detection (see below) implies that the Lyman limit must be blueward of $\sim 250 \mathrm{~nm}$, suggesting an upper limit on the redshift of $z \lesssim 1.7$. To further constrain the redshift, $z$, a power-law UV/optical spectral energy distribution (SED) with an unconstrained spectral slope, $\beta$, as well as a fixed Galactic $E(B-V)$ was assumed. The redshift was then varied and the spectrum for a given $\beta$ was modified according to the parameterization of the average Lyman absorption (Madau 1995). The magnitudes relative to each UVOT filter were then estimated for a grid of $\beta$ and $z$, with a corresponding $\chi^{2}$ value for each $\beta$-z pair. The grid of $\chi^{2}$ values were plotted to illustrate how $\beta$ and $z$ could be constrained. The probability distributions were also projected onto each axis to obtain the confidence intervals. Neither parameter is very well constrained, but the best $\beta \sim 1.93 \pm 0.22$ and $z<1.1$ at the $90 \%$ confidence level. The most likely redshift is $z=0.75$, but it is minimally peaked.
The UVOT has $U B V$ filters that approximate the Johnson system and three ultraviolet filters: UVW1 with a central wavelength of $\lambda_{c}=251 \mathrm{~nm}, \mathrm{UVM} 2$ with $\lambda_{c}=217 \mathrm{~nm}$, and UVW2 with $\lambda_{c}=188 \mathrm{~nm}$. Examination of the first settled observation (a 200 s exposure in $V$ ) revealed a new source relative to the Digital Sky Survey inside the XRT error circle. This source had a magnitude of $V=19.56 \pm 0.32$. The UVOT position of the source is R.A. $=04^{\mathrm{h}} 26^{\mathrm{m}} 28^{\mathrm{s}} 429$ and decl. $=-10^{\circ} 50^{\prime} 39^{\prime \prime} 13(\mathrm{~J} 2000.0)$, with an internal accuracy of \pm 0.01 and an absolute astrometric uncertainty of 0.56 ( $90 \%$ containment). This position is 1.3 from the reported XRT position (Pagani et al. 2006a). It is inside the XRT error circle and consistent with reported ground-based detections (Levan \& Hjorth 2006; Thöne et al. 2006). Subsequent exposures showed the source to be fading.

We performed photometry on each UVOT exposure using a circular aperture with a radius of $2^{\prime \prime}$ centered on the position of the optical afterglow. This radius is approximately equal to the FWHM of the UVOT PSF. The PSF varies with filter and with spacecraft voltage, so we did not match the extraction aperture to the PSF for each exposure. The PSF FWHM, averaged over the voltage variations, ranges from 1."79 \pm 0.05 for the $V$ filter to 2 ". $17 \pm 0.03$ for the UVW2 filter. The background was measured in a sky annulus of inner radius 17.5 and width $5^{\prime \prime}$ centered on the afterglow.

Aperture corrections were computed for each exposure to convert the $2^{\prime \prime}$ photometry to the standard aperture radii used to define UVOT's photometric zero points ( 6 " for $U B V$ and $12^{\prime \prime}$ for the UV filters). The aperture correction procedure also accommodates the variable PSF. Approximately six isolated stars were used to compute the aperture correction for each exposure. The rms scatter in the mean aperture correction for a single exposure was typically $\sim 0.07 \mathrm{mag}$. The rms scatter for each exposure was added in quadrature to the statistical error in the 2 " magnitude to obtain the total $1 \sigma$ error in each point. All detections above the $2 \sigma$ significance level are tabulated in Table 1 .

Since the UVOT is a photon-counting device, we have corrected all of our data for coincidence loss; however, the afterglow has $V>19$, so coincidence losses are negligible, typically less than 0.01 mag. The zero points used to transform the instrumental UVOT magnitudes to Vega magnitudes are listed in Table 2. They are taken from the latest in-orbit measurements as obtained from the HEASARC Swift UVOT Calibration Database (CalDB). ${ }^{13}$ Color terms were not applied to the photometric calibrations, but preliminary calibrations of on-orbit data suggest that they are negligible.

The UVOT photometry was adjusted to the $U$ band by assuming that the optical spectrum was a power law with the same

${ }^{13}$ See http://swift.gsfc.nasa.gov/docs/heasarc/caldb/swift.

TABLE 2

Zero Points Appled to the Photometry of the Afterglow of GRB 060313

\begin{tabular}{|c|c|c|}
\hline Filter & Zero Point & Error \\
\hline ......... & 17.88 & 0.09 \\
\hline$B$ & 19.16 & 0.12 \\
\hline U & 18.38 & 0.23 \\
\hline 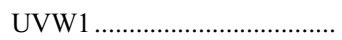 & 17.69 & 0.02 \\
\hline UVM2 & 17.29 & 0.23 \\
\hline 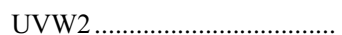 & 17.77 & 0.02 \\
\hline White & 19.78 & 0.02 \\
\hline
\end{tabular}


TABLE 3

Parameters of a Joint Bat-Konus Fit to a Model Consisting of a Power Law with an Exponential Cutoff

\begin{tabular}{|c|c|c|c|c|c|}
\hline $\begin{array}{l}t \\
\text { (s) }\end{array}$ & $f_{\mathrm{RN}}$ & $\Gamma$ & $E_{p}$ & $\chi^{2} /$ dof & Probability \\
\hline \multirow[t]{2}{*}{-0.085 to -0.107 (A) } & 1.0 & $-0.01_{-0.14}^{+0.13}$ & $844_{-95}^{+110}$ & $95.4 / 89$ & 0.30 \\
\hline & $0.80_{-0.11}^{+0.12}$ & $0.12_{-0.15}^{+0.14}$ & $831_{-97}^{+112}$ & $89.1 / 89$ & 0.45 \\
\hline \multirow[t]{2}{*}{ 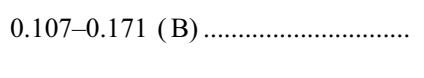 } & 1.0 & $-0.22_{-0.61}^{+0.45}$ & $237_{-58}^{+67}$ & $73.3 / 64$ & 0.20 \\
\hline & $0.96_{-023}^{+0.36}$ & $-0.23_{-0.62}^{+0.61}$ & $233_{-59}^{+98}$ & $73.2 / 63$ & 0.18 \\
\hline \multirow[t]{2}{*}{ 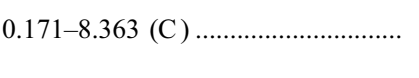 } & 1.0 & $0.80_{-0.13}^{+0.62}$ & $921_{-574}^{+49}$ & $122.4 / 125$ & 0.55 \\
\hline & $1.18_{-0.18}^{+0.16}$ & $0.74_{-0.14}^{+0.13}$ & $990_{-286}^{+521}$ & $119.8 / 124$ & 0.59 \\
\hline \multirow[t]{2}{*}{$-0.085-8.363 \ldots \ldots \ldots \ldots \ldots \ldots \ldots \ldots \ldots \ldots$} & 1.0 & $0.64_{-0.10}^{+0.08}$ & $924_{-165}^{+226}$ & $116.0 / 124$ & 0.68 \\
\hline & $1.07_{-0.12}^{+0.12}$ & $0.61_{-0.11}^{+0.09}$ & $947_{-173}^{+124}$ & $115.1 / 123$ & 0.68 \\
\hline
\end{tabular}

Notes.-Two sets of fit results are shown for each time interval $(t)$. In the first row for each time interval, the joint fitting was done without renormalization between BAT and Konus. In the second rows, the relative normalization $\left(f_{\mathrm{RN}}\right)$ is allowed to be a free parameter. In the case in which $f_{\mathrm{RN}}$ is not statistically consistent with unity, the difference is attributed to the differences in the low energy parts of the BAT and Konus spectra. The photon index is $\Gamma, E_{p}$ is the peak energy, and $\chi^{2} /$ dof is the goodness of fit.

slope as the X-ray spectrum $\left(\beta_{\mathrm{X}}=0.96\right)$. The resultant UVOT photometry is provided in Table 1 , in the "adjusted $U$-band magnitude" column, and is shown in Figure 4. In addition, the UVOT photometry was also adjusted to the $U$ band assuming the cooling break is between the optical and X-ray bands at $t>1000 \mathrm{~s}$. The assumed spectral slope in the optical is $\beta_{O}=0.46$. Although the scatter looks slightly less for the $\beta_{O}=0.46$ case, the rms residual for both cases is $0.45 \mathrm{mag}$ (see below).

As seen in Figure 4, there appears to be flaring in the UVOT light curve between 3000-40,000 s. To determine the statistical significance of these potential flares, the shifted UVOT photometry was fitted with a single power law $\left(\chi_{\text {red }}^{2}=2.59\right.$ and $\mathrm{rms}_{\text {scatter }}=0.45 \mathrm{mag}$ for $\beta_{O}=0.96 ; \chi_{\text {red }}^{2}=2.40 \& \mathrm{rms}_{\text {scatter }}=$ $0.45 \mathrm{mag}$ for $\beta_{O}=0.46$ ). Based on this fit, the fluctuations appear real. If the fluctuations represent flares and a single power law is fit to the bottom of the photometry data (i.e., a line is placed through the light curve while ignoring the three flares), the flaring becomes even more significant $\left(\chi_{\text {red }}^{2}=5.16\right.$ and $\mathrm{rms}_{\text {scatter }}=0.50 \mathrm{mag}$ for $\beta_{O}=0.96$ ). A further check was made to determine whether the fluctuations were caused by instrumental or other effects. Light curves for seven comparison stars (which were selected on the basis of similar magnitudes to the afterglow's) were constructed in each filter after shifting to the $U$ band as described above. For each comparison star a weighted mean magnitude and the residual about the weighted mean was computed. The rms residual of all the observations of all the stars is 0.09 mag, which is much smaller than the rms residual about the power-law fit to the afterglow's light curve. We therefore conclude that the comparison stars do not show the same fluctuations as the optical afterglow and that the fluctuations are intrinsic to the afterglow. Although optical flaring has been reported for long bursts (see Holland et al. 2002; Jakobsson et al. 2004), this is the first short burst with optical flaring.

\subsection{Spectral Analysis}

Joint spectral analysis was carried out using the BAT data between 15 and $150 \mathrm{keV}$ and the Konus data from 20 to $3000 \mathrm{keV}$. The spectra were fit by a power-law model with an exponential cutoff: $d N / d E \sim E^{-\Gamma} \exp ^{-(2-\Gamma) E / E_{p}}$, where $E_{p}$ is the peak energy of the $\nu F_{\nu}$ spectrum and $\Gamma$ is the photon index. A fit to the Band (GRBM) model was also attempted. No statistically significant high-energy power-law tail was established in any fitted spectrum. The time-integrated spectrum is well fit with $\Gamma=0.61 \pm$ 0.10 and $E_{p}=947_{-173}^{+224} \mathrm{keV}\left(\chi^{2}=115 / 123\right.$ degrees of freedom [dof ]). Using these parameters, the calculated burst fluence is $1.29_{-0.31}^{+0.15} \times 10^{-5} \mathrm{ergs} \mathrm{cm}^{-2}$ and the peak flux is $5.99_{-1.59}^{+0.10} \times$ $10^{-5}$ ergs cm ${ }^{-2} \mathrm{~s}^{-1}$, as measured from $T_{0}=0.475 \mathrm{~s}$ on a $16 \mathrm{~ms}$ timescale. All errors are at the $90 \%$ confidence level. Joint fits were also made to the same cutoff power-law model for three specific time intervals denoted on Figure 3. The results are summarized in Table 3. Significant spectral evolution in both the low-energy photon index and $E_{p}$ is evident.

We also constructed an SED of the optical and UV afterglow for $14 \mathrm{ks}$ after the trigger. This time was chosen because the light curve is relatively well sampled in the different UVOT bands around that point, requiring the minimum of interpolation in constructing the SED. For the $B, U$, and UVW1 bands respectively, a power law was fitted to the data points between 4000 and 40,000 s and the count rates at $14 \mathrm{ks}$ were interpolated from the fitted curves. For the $V, \mathrm{UVM} 2$, and UVW2 bands, for which there was only one point per filter in the 4000-40,000 s time range, an average power-law decay fitted jointly to $B, U$, and UVW1 was

TABLE 4

Parameters of a Power-Law Fit to the UVOT-XRT Spectrum with Galactic Dust and Gas Plus Dust Absorption Intrinsic to the GRB Host

\begin{tabular}{|c|c|}
\hline Component & Parameter Values \\
\hline Power law $(\gamma)$ & $2.03 \pm 0.04$ \\
\hline 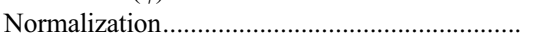 & $5.5 \pm 0.4 \times 10^{-5}$ photons $\mathrm{cm}^{-2} \mathrm{~s}^{-1} \mathrm{keV}^{-1}($ at $1 \mathrm{keV})$ \\
\hline Neutral Galactic gas absorption $\left(N_{\mathrm{H}}\right) \ldots \ldots \ldots \ldots \ldots$ & $4.74 \times 10^{20} \mathrm{~cm}^{-2}$ (fixed) \\
\hline Dust type & Milky Way \\
\hline$E(B-V)$ & $0.0625 \mathrm{mag}$ (fixed) \\
\hline$z$ & 0 (fixed) \\
\hline$\chi_{\text {red }}^{2}$ & 1.142 (34 dof) \\
\hline
\end{tabular}




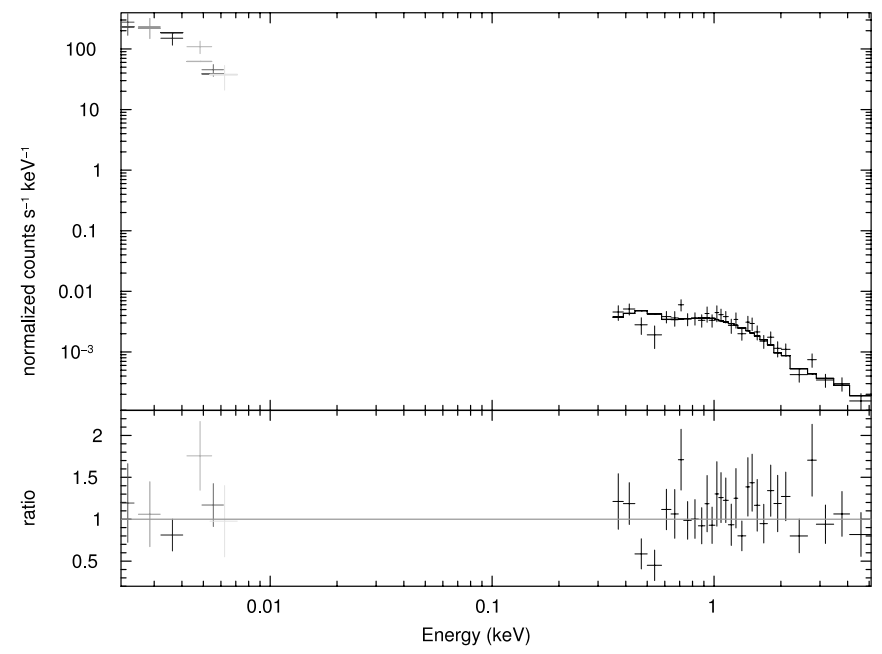

FIG. 5.- Top panel: Combined UVOT and XRT SED of the afterglow at $14 \mathrm{ks}$ after the burst. The model fit is overplotted; this model, which was fitted to both the UVOT and XRT data simultaneously, consists of a single power law with a slope of $2.03 \pm 0.04$ and Galactic neutral gas absorption and dust extinction (the full model parameters are listed in Table 4). This power law is softer that fitted to the complete XRT data set (which is biased toward earlier times due to the declining count rate). No absorption or extinction intrinsic to the GRB host is required in the fit. Bottom panel: Ratio of this model to the UVOT and XRT data. [See the electronic edition of the Journal for a color version of this figure.]

renormalized to the single points in each of $V$, UVM2, and UVW2. The count rates at $14 \mathrm{ks}$ in these filters were then determined from these extrapolated curves. The count rates from all six filters were used to make spectral files for fitting with XSPEC using the latest UVOT response matrices (version 102). The errors on the count rates consisted of a sum in quadrature of the photometric error and a $10 \%$ systematic error from the calibration uncertainty on the filter effective areas.

An XRT spectrum for the time interval 4100-24,000 s was extracted for joint fitting with the UVOT, and renormalized to the total count rate expected at $14 \mathrm{ks}$. Fitted by itself, the XRT spectrum was slightly softer than that obtained from the whole time interval, with a (Galactic absorbed) power-law slope of $1.97 \pm 0.09$. The XRT and UVOT spectra were then fitted simultaneously, with a model consisting of a power law absorbed by Galactic gas and dust as well as dust intrinsic to the GRB host.

A good fit was obtained with the power law with Galactic dust and gas alone; no dust intrinsic to the source was required. Since we cannot fit the redshift of any dust feature we are unable to use the SED fitting to constrain the redshift of the GRB in this case. The full parameters of the joint UVOT-XRT fit are given in Table 4, and the UVOT and XRT spectra are plotted with the model and the ratio of data to the model in Figure 5.

\section{DISCUSSION}

\subsection{Prompt Gamma-Ray Emission}

Although GRB 060313 is the hardest of the Swift bursts [ based on the $(50-100 \mathrm{keV}) /(25-50 \mathrm{keV})$ ratio], it is just above the average hardness for BATSE short bursts (see Fig. 6). Since the BAT light curve of GRB 060313 is bright and therefore the statistical significance is high, we can be confident that many of the multipeaked pulses in the light curve are real. Most BATSE short bursts, even when as bright as GRB 060313, did not reveal as much structure as is evident in this burst. The burst fluence as measured in the $15 \mathrm{keV}-3 \mathrm{MeV}$ band was $1.29_{-0.31}^{+0.15} \times 10^{-5} \mathrm{ergs} \mathrm{cm}^{-2}$, providing an isotropic gamma-ray energy release of $3.4 \times 10^{52} \mathrm{ergs}$ for an assumed burst of redshift 1.

The spectrum during the burst had a positive spectral index during the first $0.19 \mathrm{~s}$ of the burst $\left(f_{\nu} \propto \nu^{1.06}\right)$ and the timeaveraged low-energy spectrum was $f_{\nu} \propto \nu^{0.29 \pm 0.07}$. These spectra can be produced in the synchrotron-self-Compton model for gamma-ray generation (Kumar et al. 2006). The high degree of variability of the gamma-ray light curve is probably due to a highly variable outflow from the central explosion.

\subsection{Afterglow}

The X-ray light curve has a profile similar to the canonical profile described in the literature (Zhang et al. 2006), with the exception that segment I (the time immediately after the prompt emission) has a much shallower decay slope ( $\sim 2$ times less than the canonical). This XRT decay slope of $\alpha=1.25 \pm 0.15$, beginning $\sim 100 \mathrm{~s}$ after the burst trigger, is much less than the early decay slopes seen at similar times for some short bursts: GRBs 050724 ( $\alpha=7$; Barthelmy et al. 2005b), 050813 ( $\alpha=2.05 \pm$ 0.20 ; J. Racusin 2006, private communication), 051210 ( $\alpha=$ $2.57 \pm 0.11$; La Parola et al. 2006), and 051227 ( $\alpha=2.2 \pm 0.2$; L. M. Barbier et al. 2006, in preparation). The decay slope is more consistent with the short bursts: GRBs 050509B ( $\alpha=$ $1.20_{-0.08}^{+0.09}$; Gehrels et al. 2005), 050709 ( $\alpha \lesssim 1$; Fox et al. 2005), and 051221 A ( $\alpha=1.3 \pm 0.7$; Capalbi et al. 2005).

The initial XRT light curve has flaring in the first $1000 \mathrm{~s}$ after the trigger. The spectrum during this period is harder (photon index of $1.53 \pm 0.10$ ) than the best spectral fit of the late afterglow (photon index of $1.96 \pm 0.09$ ). Flares in the early light curve of the short GRBs 050724 (Barthelmy et al. 2005b; Campana et al. 2006; Grupe et al. 2006), 051210 (La Parola et al. 2006), and 060121 (Levan et al. 2006) have also been seen. Flaring in GRBs has been attributed to long-lasting activity by the central engine with internal shocks continuing for hundreds of seconds (Burrows et al. 2005c; Falcone et al. 2006a, 2006b; Pagani et al. 2006c; Zhang et al. 2006; De Pasquale et al. 2006). Although the decay slope of GRB 060313 is consistent with GRBs 050509B, 050709, and 051221A, no early flaring was seen in these three bursts (Fox et al. [2005] report the possibility of a flare at early times for GRB 050709; however, the fitting of the light curve at early times was difficult since only one data point was available in the first day after the burst). Between

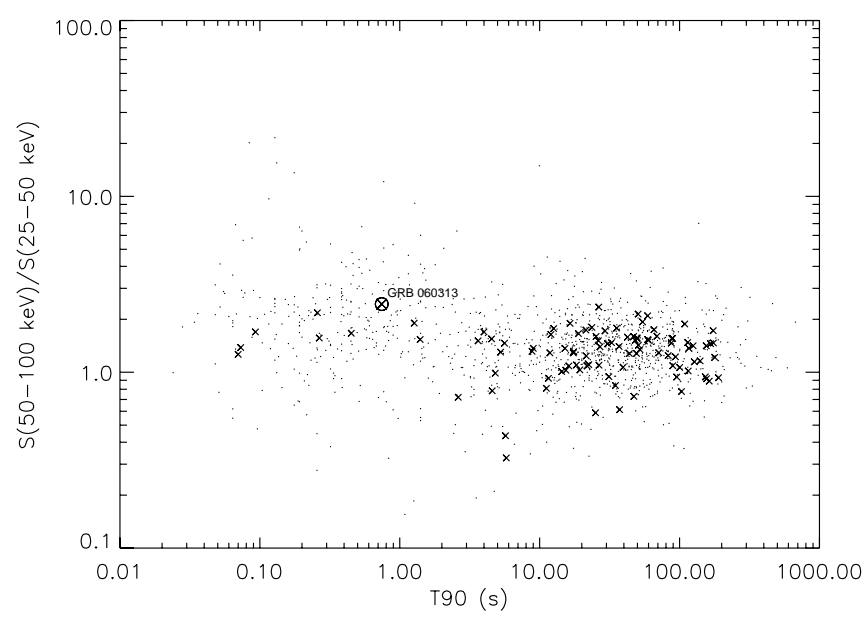

FIG. 6.- Hardness ratio vs. $T_{90}$ for the BAT and BATSE bursts. This plot shows how the spectral hardness and duration of GRB 060313 compares to previous BAT bursts (crosses), and BATSE bursts (dots). One can see that GRB 060313 is the hardest burst yet detected by BAT, although it falls well within the BATSE hardness distribution. 
1000-3000 s no data were obtained due to occultation by the Earth; therefore, it is unknown what the light-curve profile is at this time. However, this region in the light curve is consistent with either segment II or V in Zhang et al. (2006); therefore, there is clear evidence that there is some kind of energy injection

The optical and UV light curve is the earliest $(<80 \mathrm{~s})$ optical/UV detection of a short GRB to date. The early UVOT decay slope, beginning $\sim 100 \mathrm{~s}$ after the burst trigger, is $\alpha=0.13 \pm 0.28$, which is much flatter than the XRT decay slope over the same period. In addition, unlike the XRT, the UVOT light curve contains no flaring during this period. At $t>3000 \mathrm{~s}$, the UVOT light curve is steeper ( $\alpha=0.43 \pm 0.13$ ) and includes three flares superimposed on the overall light curve, while the XRT light curve has no flaring. No other short burst has so far been observed to manifest optical flaring at such early times.

The XRT spectrum during the first $1000 \mathrm{~s}$ of the burst was proportional to $\nu^{-0.53 \pm 0.10}$ in the $0.3-10 \mathrm{keV}$ energy band. The spectrum in this entire energy band was a single power law, meaning that it did not contain any characteristic synchrotron frequency. During this same period, the XRT light curve fell off as $t^{-1.25}$, signifying that the synchrotron peak frequency was below the XRT band or less than $0.3 \mathrm{keV}$. This indicates that the synchrotron cooling frequency was either less than $0.3 \mathrm{keV}$ or above $10 \mathrm{keV}$. If the cooling frequency were to be below $0.3 \mathrm{keV}$, then the XRT spectrum would be $\nu^{-p / 2}$ (where $p$ is the electron power-law distribution index), giving a value for $p=1.06 \pm 0.2$, which is highly unlikely (it is much too small for the X-ray spectrum of GRB afterglows). We therefore conclude that the cooling frequency for $t<1000 \mathrm{~s}$ was above $10 \mathrm{keV}$ (see Sari et al. 1998; Chevalier \& Li 1999; Rol et al. 2005).

An X-ray light curve due to shocks in a uniform density circumstellar medium (CSM) is expected to fall off as $t^{-0.8}$, for a photon index of 1.53 , instead of the observed $t^{-1.25}$ decay. The observed light-curve decay is consistent with a windlike density stratification for the CSM. However, the subsequent decline of the X-ray light curve and the spectrum are seriously at odds with this possibility and therefore we do not consider it to be a viable solution. The steeper-than-expected fall off of the light curve could be due to some combination of a structured jet, radiative loss of energy, and decreasing microphysics parameters $\left(\epsilon_{e}\right.$ and $\epsilon_{B}$; see Panaitescu et al. 2006).

The flux in the $0.3-10 \mathrm{keV}$ band at $100 \mathrm{~s}$ from the forward shock, assuming the XRT band below the synchrotron cooling frequency and a burst redshift of 1 , which provides a luminosity distance $\left(D_{28}\right) \sim 1$, is (eq. [B7] from Panaitescu \& Kumar (2000) is used since we assume $\nu_{i}<\nu<\nu_{c}$ and a homogeneous external medium)

$$
f_{\mathrm{X}} \approx 5 \times 10^{-9} E_{52}^{5 / 4} n^{1 / 2} \epsilon_{e,-1} \epsilon_{B,-2}^{3 / 4} \quad \operatorname{ergs~} \mathrm{cm}^{-2} \mathrm{~s}^{-1}
$$

where $E_{52}$ is the isotropic equivalent energy in units of $10^{52} \mathrm{ergs}$, $\epsilon_{e,-1}=\epsilon_{e} / 0.1\left[\epsilon_{e}(p-1) /(p-2)\right.$ is the energy fraction in electrons], $\epsilon_{B,-2}$ is the energy fraction in the magnetic field, $n$ is the density of the CSM, and we used $p=2.06$, as suggested by the early X-ray spectrum. ${ }^{14}$ in calculating the numerical values in the above equation. Note that the error in the burst redshift has a very small effect on the X-ray flux formula given above as $f_{\mathrm{X}}$ has almost a linear dependence on the isotropic burst energy and therefore the effect of uncertainty in distance on $E_{52}$ and $f_{\mathrm{X}}$ nearly

\footnotetext{
14 Since $f_{\nu} \propto \nu^{-0.53}$, then $p=2.06 \pm 0.2$. The lower bound (1.86) would lead to a negative energy fraction of electrons, which is an unphysical event.
}

cancel each other. Substituting the observed value of the X-ray flux of $3 \times 10^{-10} \mathrm{ergs} \mathrm{cm}^{-2} \mathrm{~s}^{-1}$ in the above formula, we find

$$
E_{52}^{5 / 4} n^{1 / 2} \epsilon_{e,-1} \epsilon_{B,-2}^{3 / 4} \approx 6 \times 10^{-2} .
$$

Moreover, applying the constraint that the cooling frequency is greater than $10 \mathrm{keV}$ at $10^{3} \mathrm{~s}$ (because the photon spectral index is 1.53) we obtain

$$
E_{52}^{1 / 2} n \epsilon_{B,-2}^{3 / 2}<5 \times 10^{-3} .
$$

Equation (3) follows from the expression of cooling frequency given in the literature (eq. [27] from Panaitescu \& Kumar [2000] assuming $Y \ll 1)$. Combining equations (2) and (3), we find $\epsilon_{e,-1} E_{52}>1$. Moreover, for $p=2.06$ the fraction of energy of the shocked fluid in electrons is equal to $1.76 \epsilon_{e,-1}$ which cannot be greater than 1 , and therefore $\epsilon_{e,-1}<0.6$ and $E_{52}>1.8$. The $15 \mathrm{keV}$ to $3 \mathrm{MeV}$ gamma-ray fluence suggests that the isotropic equivalent energy in photons, assuming $z=1$, was $E_{52}=3.4$, which is consistent with the above derived constraint on the energy in the explosion of $E_{52}>1.8$. Since the radiative efficiency for the prompt gama-ray emission must be less than $100 \%$, and likely of order a few tens of percent, this suggests $E_{52} \sim 6$, and $\epsilon_{e,-1} \sim 0.5$. Substituting these values in equation (2) we find

$$
n^{1 / 2} \epsilon_{B,-2}^{3 / 4} \approx 1 \times 10^{-2}
$$

We next make use of constraints on the synchrotron injection frequency (the synchrotron frequency at which the bulk of the electrons that are injected by the shock front radiate), which is given by (e.g., eq. [22] in Panaitescu \& Kumar 2000)

$$
\nu_{i}=306 E_{52}^{1 / 2} \epsilon_{e,-1}^{2} \epsilon_{B,-2}^{1 / 2} t_{2}^{-3 / 2} \mathrm{eV} .
$$

Since the XRT light curve is falling off as $t^{-1.25}$ starting from the very first XRT data point at $t_{2}=1$ (where $t_{2}=t / 100 \mathrm{~s}$ ), we conclude that $\nu_{i}<0.3 \mathrm{keV}$ at this time. If $\nu_{i}>0.3 \mathrm{keV}$, then the XRT light curve would be rising with time as $t^{1 / 2}$ (see Panaitescu $\&$ Kumar 2000). Using equation (5) for $\nu_{i}$ we therefore conclude that $\epsilon_{B,-2}<2.6 ; E_{52}=6$ and $\epsilon_{e,-1}=0.5$ were used in this estimation. Moreover, at $t=1 \mathrm{ks}$ or $t_{2}=10, \nu_{i}$ should be greater than $2 \mathrm{eV}$ since the UVOT light curve is flat during the time interval 100-1000 s. This constraint, and equation (5), leads to $\epsilon_{B,-2}>0.1$. From these upper and lower limits on $\epsilon_{B,-2}$ it seems reasonable to infer that $\epsilon_{B,-2} \sim 1$, which when substituted into equation (4) gives the density of the CSM to be $n \sim 10^{-4} \mathrm{~cm}^{-3}$. This is consistent with the combined UVOT/XRT SED, which reveals that no intrinsic dust is required to model the environment of the burst. It appears that the burst went off in a low-density medium that is usually found in bubbles or the outskirts of galaxies.

If the UVOT band lies below $\nu_{i}$, the light curve is expected to rise as $t^{1 / 2}$ (see Piran 1999; Panaitescu \& Kumar 2000). However, the light curve seems to be nearly flat for the initial $10 \mathrm{~min}$ utes or so; thus, we conclude that $\nu_{i}$ is below the UVOT band. It is likely that the same process that caused a steeper fall off of the early X-ray light curve, by $\sim t^{0.45}$, could have also flattened the rise of the early UVOT light curve from the expected $t^{1 / 2}$ to $t^{\sim 0}$. The general decline of the optical light curve between 10 and $40 \mathrm{ks}$ is roughly the same as the early X-ray light curve, and that is consistent with the forward external shock model.

The X-ray light curve for $t>4 \mathrm{ks}$ is steeper than the early light curve by $t^{1 / 4}$, and that is almost certainly a result of the cooling frequency dropping below the XRT band; the spectrum during this stage also became softer as expected for a cooling transition. 
However, the flux at $t>4 \mathrm{ks}$ is larger by a factor $\sim 4$ than the extrapolation of the earlier X-ray light curve. This suggests quite a substantial amount of energy being added to the forward shock, almost twice the amount of the initial energy in the explosion, during the time period of $1-4 \mathrm{ks}$. It is also possible that the enhanced X-ray flux could be due to the GRB ejecta running into a denser shell of CSM.

There are fluctuations in the UVOT light curve between 4 and $40 \mathrm{ks}$, whereas during this period the X-ray light curve has a smooth power-law decline. This interesting behavior is not expected for energy being added to the decelerating forward shock (due to late-time central engine activity, for instance), whereas this is consistent with fluctuations in the density of the CSM. The observed flux at frequencies above the cooling frequency $\left(\nu_{c}\right)$ is very insensitive to the CSM density structure, and this could explain the smoothly declining XRT light curve. The flux at frequencies between $\nu_{i}$ and $\nu_{c}$ varies with density as $n^{1 / 2}$ (see Piran 1999; Panaitescu \& Kumar 2000), and hence density fluctuations will be reflected in the optical light curve if $\nu_{\mathrm{opt}}\left\langle\nu_{c}\right.$; the amplitude of the UVOT flux variation corresponds to a factor of about 2 variations in CSM density, and the short timescale for the variability suggests small-scale fluctuation in the CSM. We note that the early X-ray light curve $\left(t<10^{3} \mathrm{~s}\right)$, when the XRT band was below $\nu_{c}$, also showed fluctuations, lending additional credence to the interpretation of CSM density fluctuation.

The variations in the XRT and UVOT light curve occur on very short timescales $(d t / t \ll 1$, where $d t$ is the variability timescale). These small-amplitude fluctuations can arise in the external shock with $d t / t<1$; for instance, when the shock front crosses a density clump of size much less than $r / \Gamma$ ( $r$ is the radius of the external shock and $\Gamma$ is the Lorentz factor), it will increase the observed flux a bit. This increase will last for a time $d t<t$, where $d t / t$ is determined by the clump size, and the amplitude of fluctuation by its mass.

This short burst offers conflicting evidence as to the nature of the progenitor and its surrounding medium. On the one hand, the afterglow data suggests that the burst went off in a low-density medium, and on the other hand, fluctuations in the UVOT light curve and a lack of corresponding fluctuation in the X-ray data suggest that the CSM density varied by a factor of order 2 on a length scale of a few times $10^{17} \mathrm{~cm}$.

The low density of the circumstellar medium has fluctuations that one normally associates with the surroundings of a massive star and inhomogeneities of a windy medium. However, the prospect of the progenitor being a massive star is unlikely. The mean density of the medium for this burst is small $\left(\sim 10^{-4}\right.$ particles $\mathrm{cm}^{-3}$ ) for a massive star. In addition, although it cannot be ruled out, no host galaxy was found at the location of GRB 060313. Instead, a search in the extragalactic databases indicates that there are 12 galaxies within $5^{\prime}$ of GRB 060313, of which six are within $2^{\prime}$ and one within $30^{\prime \prime}$ (B042408.28-105721.7). Could this possibly be a galaxy cluster with which the GRB is associated? Currently there are no known redshifts or color information on these galaxies; therefore, it cannot be determined for certain. However, if we assume that there is no host galaxy at the position of the GRB and that it is part of this potential galaxy cluster, it is unlikely that a massive star can travel such great distances. Massive stars live for about 10 million years, and their peculiar velocity is not more than a few tens of $\mathrm{km} \mathrm{s}^{-1}$. Therefore, in their lifetime, a massive star can only travel a distance of less than a few hundred pc.

In contrast, the natal kick of a neutron $\operatorname{star}\left(\sim 200-1000 \mathrm{~km} \mathrm{~s}^{-1}\right)$ and the typical lifetimes of compact binaries $(\sim 0.1-1 \mathrm{Gyr})$ allow for DNS and NS-BH binaries to travel great distances. Assuming a redshift for GRB 060313 of $z=0.16$ (for GRB 050709, the closest measured redshift for a short GRB; Fox et al. 2005), $z=0.35$ (the average measured redshift for short GRBs; La Parola et al. 2006), $z=0.55$ (for GRB 051221A, the farthest measured redshift for a short GRB; Soderberg et al. 2006), and $z=1.1$ (for the UVOT upper redshift limit), a compact binary progenitor could have been associated with a galaxy that is $\lesssim 6.2$, $3.5,2.7$, and 2.' 1 away, respectively. These distances are consistent with the distances to some of the galaxies in the potential cluster. Without the early light curves, particularly the UV/optical light curve, we would have automatically assumed that the progenitor of this GRB was a compact binary merger. However, the data suggests that GRB 060313 may arise from a mechanism other than the established compact binary model.

An alternative possibility is that the rapid variabilities in the UVOT light curve are produced by late-time central engine activity similar to those produced in late-time X-ray flares seen in many other GRBs (e.g., Burrows et al. 2005c; Zhang et al. 2006; Levan et al. 2006). Such a possibility has been raised to discuss other rapid optical variabilities of pre-Swift afterglows with $\Delta t<t$ (e.g., Ioka et al. 2005). In this scenario, no CBM density fluctuation is required. The lack of variability in the X-ray band may be due to the fact that the $E_{p}$ 's of these late flares are very low (i.e., in the UV/optical regime), so that their contributions to the X-ray band are negligible compared with the afterglow level. Within the late internal shock scenario, soft flares are in principle possible given the combination of a low luminosity and a large Lorentz factor as a result of less baryon loading at later times (Zhang \& Mészáros 2002). The challenge is how to restart the central engine at very late epochs in short GRBs. The same problem has also been encountered when interpreting the multiple late-time flares of GRB 050724 (Barthelmy et al. 2005b), and some suggestions have been made to explain its behavior (e.g., Barthelmy et al. 2005b; Perna et al. 2006; Proga \& Zhang 2006; Dai et al. 2006; Faber et al. 2006).

\section{CONCLUSION}

The multiwavelength light curve of the SHB GRB 060313 reveals a multipeaked structure in the gamma-ray, hard X-ray, $\mathrm{X}$-ray, and UV/optical bands at different epochs of the prompt and afterglow emissions. The hard X-ray spectrum during the prompt emission had a positive spectral index consistent with the synchrotron-self-Compton model for gamma-ray generation. The large number of peaks in the gamma-ray light curve is possibly the result of the central engine generating an extremely variable outflow.

The early (70-1000 s) X-ray temporal decay slope $(\alpha=$ $1.25 \pm 0.15)$ is exceptionally steep for the observed X-ray spectrum and manifests flaring components superimposed on the light curve. During the same period the UV/optical light curve reveals a flat decay profile $(\alpha=0.13 \pm 0.28)$ with no flaring. This behavior can be attributed to a combination of a structured jet, radiative loss of energy, and decreasing microphysics parameters. The X-ray spectrum indicates that the cooling frequency was not much greater than $10 \mathrm{keV}$ at $1000 \mathrm{~s}$ after the burst. At later times $(>3000 \mathrm{~s})$ the UV/optical light curve manifests flaring on top of a decaying profile ( $\alpha=0.43 \pm 0.13)$, while the X-ray exhibits no evidence offlaring on top of a light curve that is steeper than at earlier times. An interpretation of the data points to a burst that occurred in a low-density medium and that the CSM density varied by a factor of order 2 on a length scale of a few times $10^{17} \mathrm{~cm}$. Although the collapse of a massive star as the progenitor mechanism is doubtful (although it cannot be ruled out), the favored model of a compact binary merger also creates problems in explaining the burst's behavior. One explanation for this behavior is 
that at least some short bursts may be the result of a different mechanism other than the traditional compact binary model. An alternate explanation is that late-time central engine activity is injecting energy into the UV/optical regime but not into the X-ray regime.

This is the first short burst that has manifested this kind of behavior. Because the sample size is small, more observations of these objects are needed in order to determine whether GRB 060313 is the norm or anomalous for short GRBs. In addition, more detailed theoretical modeling is needed to establish a clearer picture of the mechanism.
This work is sponsored at Penn State by NASA contract NAS500136, at University College London, Mullard Space Science Lab and University of Leicester by funding from PPARC, and at Osservatorio Astronomico di Brera by funding from ASI on grant number I/R/039/04. The Konus-Wind experiment is supported by a Russian Space Agency contract and RFBR grant 06-02-16070. We appreciate the thorough review of this paper by an anonymous referee. We gratefully appreciate the contributions of all members of the Swift team. P. R. would like to acknowledge Heidi Roehrs for her contributions.
Aptekar, R. L., et al. 1995, Space Sci. Rev., 71, 265

Arimoto, M., et al. 2006, GCN Circ. 4550, http://gcn.gsfc.nasa.gov/gcn/gcn3/ 4550.gcn3

Barthelmy, S., Cummings, J., Gehrels, N., Krimm, H., Norris, J., Sakamoto, T. \& Palmer, D. 2006, GCN Circ. 4879, http://gen.gsfc.nasa.gov/gen/gcn3/ 4879.gcn3

Barthelmy, S. D., et al. 2005a, Space Sci. Rev., 120, 143

. 2005b, Nature, 438, 994

Berger, E., et al. 2005, Nature, 438, 988

Bloom, J. S., et al. 2006, ApJ, 638, 354

Burrows, D. N., Capalbi, M., \& Grupe, D. 2005a, GCN Circ. 4366, http:// gcn.gsfc.nasa.gov/gcn/gcn3/4366.gcn3

Burrows, D. N., et al. 2005b, Space Sci. Rev., 120, 165

. 2005c, Science, 309, 1833

2006, ApJ, in press (astro-ph/0604320)

Campana, S., et al. 2006, A\&A, 454, 113

Capalbi, M. Perri, M., Burrows, D. N., Grupe, D., Boyd, P., \& Voges, W. 2005, GCN Circ. 4374, http://gcn.gsfc.nasa.gov/gcn/gcn3/4374.gcn3

Chevalier, R. A., \& Li, Z.-Y. 1999, ApJ, 520, L29

Cline, D. B., Czerny, B., Matthey, C., Janiuk, A., \& Otwinowski, S. 2005, ApJ, 633, L73

Covino, S., et al. 2006, A\&A, 447, L5

Dai, Z. G., Wang, X. Y., Wu, X. F., \& Zhang, B. 2006, Science, 311, 1127

De Pasquale, M., et al. 2006, MNRAS, 370, 1859

Dickey, J. M., \& Lockman, F. J. 1990, ARA\&A, 28, 215

Eichler, D., Livio, M., Piran, T., \& Schramm, D. N. 1989, Nature, 340, 126

Faber, J. A., Baumgarte, T. W., Shapiro, S. L., \& Taniguchi, K. 2006, ApJ, 641, 93

Falcone, A. D., et al. 2006a, ApJ, 641, 1010

2006b, ApJ, in AIP Conf. Proc. 836, Gamma-Ray Burst in the Swift

Era, ed. S. S. Holt, N. Gehrels, \& J. A. Nousek (Melville: AIP), 386

Fishman, G. J., et al. 1994, ApJS, 92, 229

Fox, D. B., et al. 2005, Nature, 437, 845

Fryer, C. L., Woosley, S. E., \& Hartmann, D. H. 1999, ApJ, 526, 152

Gehrels, N., et al. 2004, ApJ, 611, 1005

2005, Nature, 437, 851

Golenetskii, S., Aptekar, R., Mazets, E., Pal'shin, V., Frederiks, D., \& Cline, T. 2006, GCN Circ. 4881, http://gcn.gsfc.nasa.gov/gcn/gcn3/4881.gcn3

Grupe, D., Burrows, D. N., \& Patel, S. 2005, GCN Circ. 4389, http://gen.gsfc .nasa.gov/gcn/gcn3/4389.gen3

Grupe, D., Burrows, D. N., Patel, S. K., Kouveliotou, C., Zhang, B., Mészáros, P., Wijers, R. A. M., \& Gehrels, N. 2006, ApJ, in press (astro-ph/0603773)

Hill, J., et al. 2004, Proc. SPIE, 5165, 217

Holland, S. T., Barthelmy, S., Beardmore, A., Gehrels, N., Kennea, J., Page, K., Palmer, D., \& Rosen, S. 2005, GCN Circ. 4034, http://gcn.gsfc.nasa.gov/ $\mathrm{gcn} / \mathrm{gcn} 3 / 4034 . \mathrm{gcn} 3$

Holland, S. T., et al. 2002, AJ, 124, 639

Horváth, I. 1998, ApJ, 508, 757

Horváth, I., Balázs, L. G., Bagoly, Z., Ryde, F., \& Mészáros, A. 2006, A\&A, 447, 23

Hurley, K., et al. 2005, ApJS, 156, 217

Ioka, K., Kobayashi, S., \& Zhang, B. 2005, ApJ, 631, 429

Jakobsson, P., et al. 2004, NewA, 9, 435

Kouveliotou, C., Meegan, C. A., Fishman, G. J., Bhat, N. P., Briggs, M. S., Koshut, T. M., Paciesas, W. S., \& Pendleton, G. N. 1993, ApJ, 413, L101

Krimm, H., et al. 2005, GCN Circ. 3926, http://gcn.gsfc.nasa.gov/gen/gcn3/ 3926.gcn3

Kumar, P., McMahon, E., Barthelmy, S. D., Burrows, D., Gehrels, N., Goad, M.,

Nousek, J., \& Tagliaferri, G. 2006, MNRAS, 367, L52

La Parola, V., et al. 2006, A\&A, 454, L753
Lattimer, J. M., \& Schramm, D. N. 1976, ApJ, 210, 549

Lee, W. H., Ramirez-Ruiz, E., \& Granot, J. 2005, ApJ, 630, L165

Levan, A. J., \& Hjorth, J. 2006, GCN Circ. 4871, http://gcn.gsfc.nasa.gov/gen/ $\operatorname{gcn} 3 / 4871 . \mathrm{gcn} 3$

Levan, A. J., et al. 2006, ApJ, 648, L9

Madau, P. 1995, ApJ, 441, 18

Markwardt, C., et al. 2006, GCN Circ. 4873, http://gcn.gsfc.nasa.gov/gcn/ gcn3/4873.gcn3

Mineo, T., et al. 2005, GCN Circ. 4188, http:/gcn.gsfc.nasa.gov/gen/gcn3/ 4188.gen3

Mochkovitch, R., Hernanz, M., Isern, J., \& Martin, X. 1993, Nature, 361, 236

Morris, D. C., Burrows, D. N., Kennea, J. A., Racusin, J. L., Cucchiara, N., Retter, A., \& Gehrels, N. 2005, GCN Circ. 3790, http://gcn.gsfc.nasa.gov/ $\mathrm{gcn} / \mathrm{gcn} 3 / 3790 . \mathrm{gcn} 3$

Mukherjee, S., Feigelson, E. D., Jogesh Babu, G., Murtagh, F., Fraley, C., \& Raftery, A. 1998, ApJ, 508, 314

Narayan, R., Paczyński, B., \& Piran, T. 1992, ApJ, 395, L83

Norris, J. P., \& Bonnell, J. T. 2006, ApJ, 643, 266

Paczyński, B. 1986, ApJ, 308, L43 1991, Acta Astron., 41, 257

Pagani, C., Morris, D., Grupe, D., \& Burrows, D. 2006a, GCN Circ. 4875, http://gcn.gsfc.nasa.gov/gcn/gcn3/4875.gcn3

Pagani, C., et al. 2006b, GCN Circ. 4867, http://gcn.gsfc.nasa.gov/gen/gen3/ 4867.gcn3

. 2006c, ApJ, 645, 1315

Panaitescu, A. 2006, MNRAS, 367, L42

Panaitescu, A., \& Kumar, P. 2000, ApJ, 543, 66

Panaitescu, A., Mészáros, P., Burrows, D. N., Nousek, J. A., Gehrels, N. O’Brien, P., \& Willingale, R. 2006, MNRAS, 369, 2059

Parsons, A., et al. 2005, GCN Circ. 4363, http://gen.gsfc.nasa.gov/gcn/gcn3/ 4363.gen3

Perna, R., Armitage, P. J., \& Zhang, B. 2006, ApJ, 636, L29

Piran, T. 1999, Phys. Rev., 314, 575

Proga, D., \& Zhang, B. 2006, MNRAS, 370, L61

Retter, A., et al. 2005, GCN Circ. 3788, http://gcn.gsfc.nasa.gov/gcn/gcn3/ 3788.gcn3

Rol, E., Wijers, R. A. M. J., Kouveliotou, C., Kaper, L., \& Kaneko, Y. 2005, ApJ, 624, 868

Roming, P., Breeveld, A. A., Marshall, F., Parsons, A., Fink, R., \& Ajello, M 2005a, GCN Circ. 4390, http://gcn.gsfc.nasa.gov/gcn/gcn3/4390.gcn3

Roming, P. W. A., et al. 2005b, Space Sci. Rev., 120, 95

Rosswog, S., Ramirez-Ruiz, E., \& Davies, M. B. 2003, MNRAS, 345, 1077

Sakamoto, T., et al. 2006, in AIP Conf. Proc. 836, Gamma-Ray Bursts in the Swift Era, ed. S. S. Holt, N. Gehrels, \& J. A. Nousek (Melville: AIP), 43

Sari, R., Piran, T., \& Narayan, R. 1998, ApJ, 497, L17

Soderberg, A. M., \& Frail, D. A. 2006, GCN Circ. 4884, http://gcn.gsfc .nasa.gov/gcn $/ \mathrm{gcn} 3 / 4884 . \mathrm{gcn} 3$

Soderberg, A. M., et al. 2006, ApJ, in press (astro-ph/0601455)

Thöne, C., Feron, C., Hjorth, J., \& Jensen, B. L. 2006, GCN Circ. 4874, http:// gen.gsfc.nasa.gov/gcn $/ \mathrm{gcn} 3 / 4874 . \mathrm{gcn} 3$

Tueller, J., et al. 2005, GCN Circ. 3005, http:/gcn.gsfc.nasa.gov/gcn/gcn3/ 3005. $\operatorname{gcn} 3$

Vaughan, S., et al. 2006, ApJ, 639, 323

Villasenor, J. S., et al. 2005, Nature, 437, 855

Zhang, B., Fan, Y. Z., Dyks, J., Kobayashi, S., Mészáros, P., Burrows, D. N., Nousek, J. A., \& Gehrels, N. 2006, ApJ, 642, 354

Zhang, B., \& Mészáros, P. 2002, ApJ, 581, 1236

Note added in proof.- - Recent work by J. Grindlay, S. Portegies Zwart, \& S. McMillan (Nature Phys., 2, 116) does not agree that the formation of a DNS system imparts large kicks. They invoke globular clusters having an in situ origin in galaxy halos where short GRB DNS systems reside, rather than the old argument that they were kicked out there. 